\title{
Reachability problems in low-dimensional iterative maps
}

\author{
Oleksiy Kurganskyy $^{1}$, Igor Potapov ${ }^{2}$, and Fernando Sancho Caparrini ${ }^{3}$ \\ 1 Institute of Applied Mathematics and Mechanics, Ukrainian National Academy of \\ Sciences, 74 R. Luxemburg St, Donetsk, Ukraine, kurgansk@gmx.de \\ 2 Department of Computer Science, University of Liverpool, Chadwick Building, \\ Peach St, Liverpool L69 7ZF, U.K., igor@csc.liv.ac.uk \\ 3 Department of Computer Science and Artificial Intelligence, University of Seville, \\ Spain, fsancho@us.es
}

\begin{abstract}
In this paper we analyse the dynamics of one-dimensional piecewise maps (PAMs). We show that one-dimensional PAMs are equivalent to pseudo-billiard or so called "strange billiard" systems. We also show that the more general class of rational functions leads to undecidability of reachability problem for one-dimensional piecewise maps with a finite number of intervals.
\end{abstract}

Keywords: Theory of computing, iterative piecewise maps, pseudo-billiard systems, universality

\section{Introduction}

In the present work we investigate a class of hybrid systems defined by onedimensional piecewise maps. We are mainly interested in a class of one-dimensional piecewise-affine maps (PAMS) [2]. The analysis of piecewise-affine maps is one of the simplest model that generate complex behaviour, see $[2-4,6,7]$. It is known that the reachability problem is undecidable for the two-dimensional case and it is open for dimension one $[1,2]$.

It was recently shown that PAM is equivalent to hierarchical piecewise constant derivatives system (HPCD)[2]. In this paper we show that PAM is also equivalent to planar pseudo-billiard systems (PBSs) or so called "strange billiards" model that is a well known object in bifurcation and chaos theory [10, 11]. In contrast to HPCD which is a hybrid automaton where each state is defined by planar piecewise constant derivatives system (PCD), the model of PBS can also be seen as two dimensional linear hybrid automaton but with only one state.

Although the reachability for PAMs is known to be open we think that the shown equivalence between PBSs and PAMs can be useful and the results from chaos theory about "strange billiards" $[10,5,11]$ could help understand the complexity in one-dimensional piecewise-affine maps. 
In the second part of this paper we are exploring the complexity of more general class of rational maps that includes affine maps. It was shown in [8] that piecewise iterative maps defined by a very restricted basis of elementary functions:

$$
\left\{x^{2}, x^{3}, \sqrt[2]{x}, \sqrt[3]{x}, x \pm 1,10 \cdot x\right\}
$$

can simulate a Minsky machine even in dimension one. Comparing to [8], we found a new way how to create a copy of information for a temporal use in dimension one. We show that it is possible to avoid square root and cube root functions using only rational functions. However in the current construction two of the finite number of intervals we define are infinite. As a main result in this part we show how to simulate (in direct way) a Minsky machine in one-dimensional piecewise rational maps (PRM) of degree 2. From it follows that the reachability problem for PRM is undecidable.

It would be interesting to investigate a natural class of one-dimensional piecewise linear rational maps that is in between affine and rational maps. The main motivation for this class of systems is based on the fact that the reachability in one-dimensional piecewise linear rational maps can be seen as parameterized reachability in two dimensional linear ${ }^{4}$ maps. Another interesting question is nondeterministic maps where transformations can be applied in any order. In this case reachability problems for nondeterministic linear rational maps corresponds to parametrized membership in $2 \times 2$ matrix semigroups. According to undecidability in PRM we think that it is also very likely to find undecidability of reachability problems in a nondeterministic version of one-dimensional rational maps.

\section{Preliminaries}

In what follows we use traditional denotations $\mathbb{N}, \mathbb{Q}$ and $\mathbb{R}$ for the sets of naturals, rationals and reals respectively.

A function from a set $A$ to a set $B$, we will denote by $f: A \rightarrow B$. If $f$ is an injection such that $\operatorname{dom}(f)=A$, then it will be denoted by $f: A \hookrightarrow B$. In some cases we put $x \mapsto y$ under the definition of a function $f$ to express that $y=f(x)$, for example:

$$
\begin{aligned}
f: & \mathbb{R} \rightarrow \mathbb{R} \\
x & \mapsto a x+b
\end{aligned}
$$

is a way to say that $f(x)=a x+b$.

If we have a set $A$ in a topological space (usually we will consider $\mathbb{R}$ or $\mathbb{R}^{2}$ with the euclidian topology), we will denote by $\operatorname{int}(A)$ (the interior of $A$ ) the greatest open subset of $A(\operatorname{int}(A)=\cup\{G: G$ open and $G \subseteq A\})$.

\footnotetext{
${ }^{4}$ A two dimensional linear function $f$ is a function of the following type $f(x, y)=$ $a x+b y$
} 


\subsection{Dynamical Systems}

Definition 1. A dynamical transition system is a triple $S=(X, T, \Sigma)$, where $X$ is a set (the set of points of the system), $T: X \rightarrow X$ (the transition function that produces the evolution of the system), and $\Sigma$ is a collection of subsets of $X$ (this component is only considered in the case we are interested in the symbolic behavior of the system).

Remark 1. Usually, we will require $\Sigma$ to be a partition of $X$, or at least to be a collection of pairwise disjoint subsets of $X$ (in the case we are interested in the dynamical behavior of some parts of $X$, using the rest as auxiliary computation). Also, we will see $\Sigma$ as an alphabet, and we will study the language generated by the system on this alphabet.

Definition 2. Let $S=(X, T, \Sigma)$ a dynamical system, and $x \in X$. The sequence $\left\{x_{n}\right\}_{n \geq 0}$, such that:

$-x_{0}=x$,

- for every $n \geq 0, x_{n+1}=T\left(x_{n}\right)$.

is called the orbit of $x$ by the system $S$, and it will be denoted as $O_{S}(x)$.

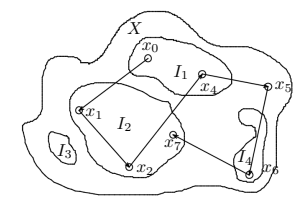

Fig. 1. Example of a dynamical system with an orbit in it

In Figure 1 we have a dynamical system $\left.S=(X, T, \Sigma)=\left\{I_{1}, I_{2}, I_{3}, I_{4}\right\}\right)$, where a partial orbit of a point, $x_{0}$, is shown.

Definition 3. Let $S=(X, T, \Sigma)$ be a dynamical system, and $x \in X$. Let's associate the set $X \backslash \cup_{w \in \Sigma} w$ to the element $\varepsilon$ (the empty word). The symbolic dynamics of $x$ in terms of $\Sigma$ is the set:

$$
\mathcal{S}_{S}(x)=\left\{w \in \Sigma^{*}: \forall n \geq 0\left(O_{S}(x)_{n} \in w_{n}\right)\right\}
$$

Where we use the notation $w=w_{1} w_{2} \ldots$

In example above, $\mathcal{S}_{S}\left(x_{0}\right)=I_{1} I_{2} I_{2} I_{1} \varepsilon I_{4} I_{2}=I_{1} I_{2} I_{2} I_{1} I_{4} I_{2}$. Note that point $x_{5}$ in the orbit has no representation in its symbolic dynamics.

Remark 2. If $\Sigma$ is a collection of pairwise disjoint subsets of $X$, then for every point $x \in X, \mathcal{S}_{S}(x)$ has only one element. 
Definition 4. Let $S_{1}=\left(X_{1}, T_{1}, \Sigma_{1}\right)$ and $S_{2}=\left(X_{2}, T_{2}, \Sigma_{2}\right)$ two dynamical systems. We will say that $S_{2}$ simulates $S_{1}$ if there exists an injection $\varphi: X_{1} \hookrightarrow X_{2}$, and an injection $\sigma: \Sigma_{1} \hookrightarrow \Sigma_{2}$ such that for every $x \in X_{1}$, we have:

$$
\mathcal{S}_{S_{2}}(\varphi(x))=\widehat{\sigma}\left(\mathcal{S}_{S_{1}}(x)\right)
$$

where $\widehat{\sigma}: \Sigma_{1}^{*} \hookrightarrow \Sigma_{2}^{*}$ is the morphism generated by $\sigma$.

\subsection{Pseudo Billiard Systems}

Let us introduce the pseudo billiard model that already appeared in a different context and became an abstract framework for some practical problems. In this system we consider a number of segments with vector fields assigned to them. The computation in this system can be described by the dynamics of the particle, which initially moves with the constant velocity (in a particular direction) inside a given region (not necessarily a polyhedron) and changes it instantaneously at the moment of a collision with the boundary to the velocity defined by a given vector field on the boundary.

We start with a more general definition for PBS's, where we have no constraints on distributing the segments around the space. In this case, a particle can touch the segments by both faces, and therefore it may cross them by the action of their projection vectors.

Definition 5. A Pseudo Billiard System $(P B S)$ is a pair $(\mathcal{A}, \mathcal{V})$, where $\mathcal{A}$ is a set of pairwise disjoint segments in $\mathbb{R}^{2}$ (closed, open or semi-open), and $\mathcal{V}=$ $\left\{\boldsymbol{v}_{A}\right\}_{A \in \mathcal{A}}$ is a set of vectors in $\mathbb{R}^{2}-\{(0,0)\}$ ( $\boldsymbol{v}_{A}$ is called the projection vector of $A$ ).

The dynamics of a particle in PBS can be defined as follows. Let a particle $P$ that is represented by a vector $x$ and is located on a segment $A \in \mathcal{A}$, i.e. $x \in A$. The transition function that moves $\mathrm{P}$ from $x$ to a position $x^{\prime}$ can be defined as follows: $x^{\prime}=x+\lambda \boldsymbol{v}_{A}$, where $\lambda=\min \left\{\delta>0: x+\delta \boldsymbol{v}_{A} \in \bigcup_{A^{\prime} \in \mathcal{A}} A^{\prime}\right\}$. We will suppose that for every $x \in A$ there exists such a $\lambda$ (the particle is trapped inside the system).

a)

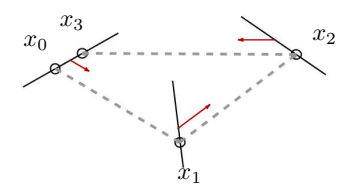

b)

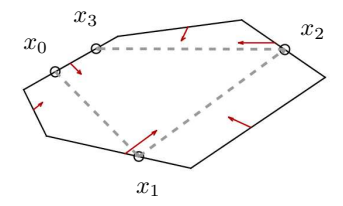

Fig. 2. An example of partial orbit: a) in a PBS, b) in a reflecting PBS

The PBS can be seen as a dynamical transition system $S=(X, T, \Sigma)$ where: 
- $X=\bigcup_{A \in \mathcal{A}} A$

$-T(x)=x+\lambda \boldsymbol{v}_{A}$, where $x \in A$ and $\lambda=\min \left\{\delta>0: x+\delta \boldsymbol{v}_{A} \in \bigcup_{A^{\prime} \in \mathcal{A}} A^{\prime}\right\}$

$-\Sigma$ is any collection of subsets of $X$ (usually it will be a subset of $\mathcal{A}$ ).

Definition 6. $A P B S$ is reflecting, if for every $A \in \mathcal{A}$, the set $T^{-1}(A)$ and $T(A)$ are in the same half-plane determined by $A$.

\subsection{Piecewise Affine Maps}

Definition 7. We say that $f: \mathbb{R} \rightarrow \mathbb{R}$ is a piecewise affine map (PAM) if there exists a partition of dom $(f)$ in a finite number of intervals of $\mathbb{R}$ (we allow the intervals to be closed, open or semi-open intervals), $\mathcal{I}$, and for every $I \in \mathcal{I}$, there exists $a_{I}, b_{I} \in \mathbb{R}$ such that $\forall x \in I, f(x)=a_{I} x+b_{I}$.

Remark 3. If we have $f(\operatorname{dom}(f)) \subseteq \operatorname{dom}(f)$, then we can consider a dynamical system associated to it, $S=(X, T, \Sigma)$ where:

$-X=\operatorname{dom}(f)$,

$-T=f(x)$ and

$-\Sigma$ is any collection of subsets of $X$ (usually it will be a subset of $\mathcal{I}$ ).

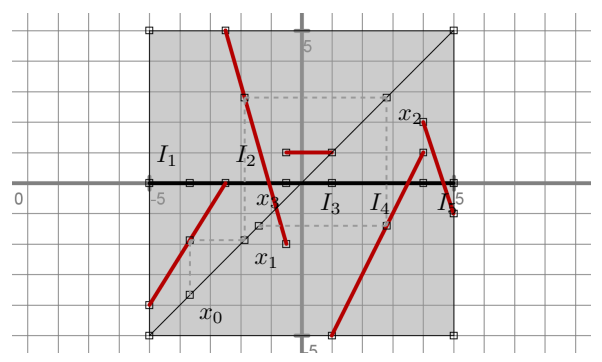

Fig. 3. An example of partial orbit in a PAM (represented on the diagonal)

Definition 8. A rational function is a function defined as a ratio of polynomials. For a single variable $x$ a typical rational function is therefore $f(x)=$ $P(x) / Q(x)$, where $P$ and $Q$ are polynomials in $x$ as indeterminate, and $Q$ is not the zero polynomial.

We also give the definition of a more general class of rational functions that we are going to study in the paper. We define it over $\mathbb{Q}$ to show that even in this case the predictability of its behaviour is an undecidable problem.

Definition 9. A Piecewise (one-dimensional) rational map (PRM) is a function that is defined on a finite sequence of disjoint intervals $I_{-}=\left(-\infty, r_{-}\right], I_{+}=$ $\left[l_{+},+\infty\right), I_{i}=\left[l_{i}, r_{i}\right]$ with $r_{-}, l_{+}, l_{i}, r_{i} \in \mathbb{Q}, i=1 . . k$ and uses rational functions for different parts of its domain. 
The computation in the above system can be understood as a generation of sequence of points. One of the obvious problems that arises in such systems is a point-to-point reachability problem that can be formulated as follows:

Problem 1. Given two points $x, y \in \mathbb{Q}$ and a one-dimensional piecewise map $P$. Decide whether $y$ is reachable from $x$ in $P$.

\section{Equivalence between Dynamical Systems}

In this section we will study the equivalence between the models introduces above. We will say that two models are equivalent if for every system of one type there exists a system of another type that simulates it and vice versa. In particular we are giving geometrical constructions to show the equivalence of onedimensional PAM, planar PBS and planar reflective PBS. Moreover using the result that model of hierarchical piecewise constant derivative systems (HPCDs) is equivalent to one-dimensional PAMs we can state that planar PBS is equivalent to two-dimensional HPCDs (see [2]). Hence the complexity that can be obtained with any of them is the same.

\subsection{PAM simulates PBS}

The first step through the equivalence will be devoted to prove that any PBS system (reflecting or not) can be simulated by a PAM system.

Theorem 1. For every Pseudo Billiard System, $\{\mathcal{A}, \mathcal{V}\}$, there exists a Piecewise Affine Map that simulates it.

Proof. Let us consider a PBS given by a set of segments $\mathcal{A}=\left\{A_{i}\right\}$ and a set of associated projection vectors $\left\{\boldsymbol{v}_{i}\right\}$. You can see an example on Figure 4 .a.

a)

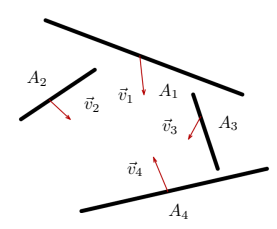

b)

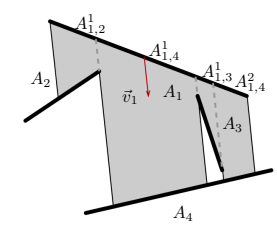

Fig. 4. a) Example of PBS to be simulated by a PAM; b) Projection of $A_{1}$ on other segments of the PBS, and the partition on $A_{1}$ that it generates

The dynamics of the PBS is defined by projecting every point of $A_{i}$ on some other segment by using the projection vector $\boldsymbol{v}_{i}$. It is clear, from the definition of PBS, that we can make a partition of $A_{i}$ in segments, $\left\{A_{i, j}^{k}\right\}$, in such a way that every point of $\left\{A_{i, j}^{k}\right\}$ is projected on a point of $A_{j}$ (see Figure 4 .b). The next step is to associate for every segment of the system, $A_{i}$, an interval on 
the line, $I_{i}$, by using an affine bijection, $\mu_{i}: A_{i} \rightarrow I_{i}$. Also, we will require these intervals to be pairwise disjoint. For every $i, j, k$ such that $A_{i, j}^{k} \neq \emptyset$, the projection $P_{i, j}^{k}: A_{i, j}^{k} \rightarrow A_{j}$ is an affine transformation. Also, all the functions $\mu_{i}$ are affine transformations.

a)

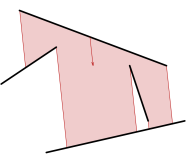

b)

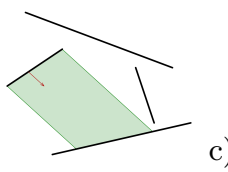

d)

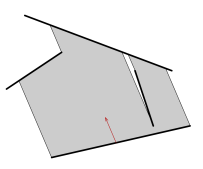

Fig. 5. Projections: a) from $A_{1} ;$ b) from $A_{2} ;$ c) from $A_{3}$; d) from $A_{4}$

Hence, we can define an affine map

$$
\begin{aligned}
f_{i, j, k}: \mu_{i}\left(A_{i, j}^{k}\right) & \rightarrow \mu_{j}\left(A_{j}\right) \\
x & \mapsto \mu_{j}\left(P_{i, j}^{k}\left(\mu_{i}^{-1}(x)\right)\right)
\end{aligned}
$$

Since $\left\{A_{i, j}^{k}\right\}_{i, j, k}$ is a partition of the points of the PBS, $\left\{I_{i, j}^{k}\right\}_{i, j, k}$ is a partition of the set of intervals considered, hence we obtain that the map

$$
\begin{aligned}
f: \bigcup_{i, j, k} I_{i, j}^{k} & \rightarrow \bigcup_{i, j, k} I_{i, j}^{k} \\
x & \mapsto f_{i, j, k}(x), \text { if } x \in I_{i, j}^{k} \text { is a piecewise affine map. }
\end{aligned}
$$

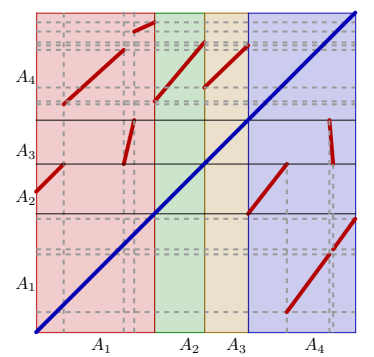

Fig. 6. PAM obtained after the process

In order to prove the dynamical simulation, let us consider the following injection between the set of points of the systems:

$$
\begin{aligned}
\varphi: \bigcup_{x} A_{i} & \hookrightarrow \bigcup I_{i} \\
& \mapsto \mu_{i}(x), \text { if } x \in A_{i} .
\end{aligned}
$$

If $\Sigma$ is the subset of $A$ that produces the symbolic dynamics, then $\Sigma^{\prime}=$ $\{\varphi(I): I \in \Sigma\}$ is the collection to be considered in the PAM, and $\sigma: \Sigma \rightarrow \Sigma^{\prime}$ 
defined by $\sigma(I)=\varphi(I)$ is the injection for the dynamics. From the construction, it is obvious that $f$ simulates the given PBS.

Lemma 1. The number of affine functions we need in order to simulate a PBS is bounded ${ }^{5}$ by $|\mathcal{A}|(|\mathcal{A}|+2)$.

Proof. Idea: for every segment of the PBS, the partition we need to make all possible projections on the other segments is bounded in size by $|\mathcal{A}|+2$.

\subsection{PBS simulates PAM}

Next, we will prove that for any PAM we can build a PBS simulating its dynamical behavior. Indeed, we will see that they can be simulated using only reflecting PBS's, hence there is no difference (regarding the dynamical complexity of the system) in using a general PBS or restrict ourselves to reflecting PBS's.

Nevertheless, we will prove in a first step that, for every PAM we can get a general PBS (usually not reflecting) that simulates the given PAM. The proof is based on the graphical idea about how to compute the orbit of a point directly on the graph generated by the PAM (where we consider the dynamics on the diagonal rather than on the $X$ axis) using the iterated projections between the affine map and the diagonal.

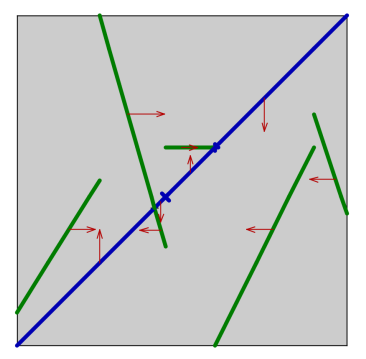

Fig. 7. PBS associated to a PAM

In this example we can see that the PBS obtained by the dynamics of the PAM over the diagonal is, in general, a non reflecting one, because depending its definition, we will need to cross some of the affine map graphs to reach the diagonal. In any case, it is not a problem, because if we must cross one map from another one, it is because both of them are in the same half-plane from the diagonal, and then their associated vectors are parallel and in the same direction.

It is easy to see that, if we restrict the dynamics on the set of segments over the diagonal, rather than on the $X$ axis, the system we obtain is equivalent to the original one.

\footnotetext{
${ }^{5}$ In case of reflecting PBSs, the bound can be reduced to $|\mathcal{A}|+2$
} 
Theorem 2. For every Piecewise Affine Map there exists a Pseudo Billiard System that simulates it.

Proof. Let $f=\bigcup_{i=1}^{n} f_{i}$ a PAM where every $f_{i}$ is an affine map over an interval $I_{i}$. We consider the following segments on $\mathbb{R}^{2}$ :

- For every $I_{i}=\left[a_{i}, b_{i}\right]$, we consider its projection on the diagonal, $x=y$, that we note as $A_{i}$.

- For every $I_{i}$ we consider the segment given by $\left(a_{i}, f_{i}\left(a_{i}\right)\right)-\left(b_{i}, f_{i}\left(b_{i}\right)\right)$, that we note as $f\left(A_{i}\right)$.

We can consider that there is no intersection between the interior of segments of the PAM (otherwise if any $A_{i}$ intersects with some $f\left(A_{j}\right)$, we consider the intersection point, and subdivide both segments, leaving this point in the diagonal segment (in Figure 7 we have split the second affine function in order to have segments with no intersection in their interiors). Of course, the obtained $\mathrm{PAM}$ is equivalent to the original one.

The vectors associated with every segment is given by the following rule: for every $i$

- if $\operatorname{int}\left(f\left(A_{i}\right)\right)$ is inside the half-plane $x<y$ (the upper half) then the vector associated to $A_{i}$ is $(0,1)$, and the vector associated to $f\left(A_{i}\right)$ is $(1,0)$.

- if $\operatorname{int}\left(f\left(A_{i}\right)\right)$ is inside the half-plane $x>y$ (the lower half) then the vector associated to $A_{i}$ is $(0,-1)$, and the vector associated to $f\left(A_{i}\right)$ is $(-1,0)$.

In order to prove the dynamical simulation, let us consider the following injection between the set of points of the systems:

$$
\begin{aligned}
\varphi: \bigcup_{x} I_{i} & \hookrightarrow \bigcup A_{i} \\
& \mapsto(x, x)
\end{aligned}
$$

and, following the same procedure as in theorem 1, the same injection between the dynamics of the systems.

From the construction, it is obvious that the resulting PBS simulates the given PAM (note that we use only a part of the dynamics of the PBS, considering only the dynamics on the diagonal, and not the evolution of the points through the other segments, necessary for the correct computing of the evolution, but not for the dynamics itself).

\subsection{Reflecting PBS simulates PAM}

Theorem 3. For every Piecewise Affine Map there exists a Reflecting Pseudo Billiard System that simulates it.

Proof. Let $f: I \rightarrow I$ be a PAM expressed in such a way that $I=\bigcup_{i=1}^{n} I_{i}$ is union of pairwise disjoint intervals, and for every $i, f_{\mid I_{i}}=f_{i}$, where $f_{i}(x)=a_{i} x+b_{i}$ is an affine function.

The first step of the proof consists in assigning to every interval of the PAM a segment in $\mathbb{R}^{2}$ where we simulate the dynamics of the system. Since $f_{i}: I_{i} \rightarrow I$ 


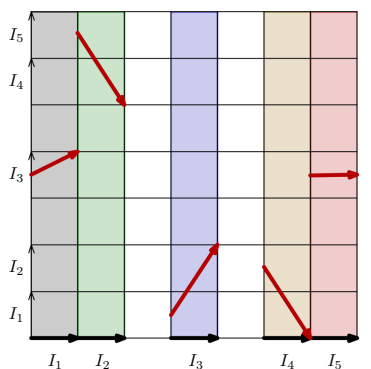

Fig. 8. PAM to be simulated

is affine, and $I_{i}$ is an interval, $f_{i}\left(I_{i}\right)$ must be an interval too. Hence, the image of every interval of our partition must be inside an union of intervals of our partition that constitutes a larger interval. To make more direct the proof, we will maintain the continuity among intervals of $f$ by considering for every interval, $I_{i} \subseteq \mathbb{R}$, of $f$, the segment $A_{i}=I_{i} \times\{0\} \subseteq \mathbb{R}^{2}$.

Now, we will simulate the dynamics of each affine map separately. Because the segments $A_{i}$ are in the same line, we can't go directly from one to another by using projections, therefore we will make use of auxiliary reflection segments to produce the same result as $f$ produces.

Depending on the coefficients of the affine map, there are three different cases:

1. Case 1: $a_{i}>0$. In this case there is no flip from $A_{i}$ to $f_{i}\left(A_{i}\right)$, so we will need only one reflecting auxiliary segment to simulate the application of $f$, $B_{i}$ (see figure 9 .a).

a)

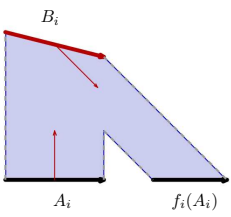

b)

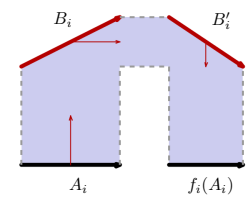

c)

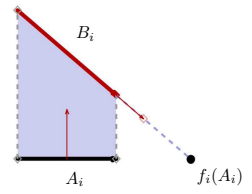

Fig. 9. a) Case 1: $a_{i}>0$ b) Case 2: $a_{i}<0$ c) Case 3: $a_{i}=0$

2. Case 2: $a_{i}<0$. In this case there is a flip from $A_{i}$ to $f_{i}\left(A_{i}\right)$, so we will need two reflecting auxiliary segments, $B_{i}$ and $B_{i}^{\prime}$, to simulate the application of $f$ (see figure $9 . \mathrm{b})$.

3. Case 3: $a_{i}=0$. In this case $f\left(A_{i}\right)$ is a point, and we will make use of only one reflecting auxiliary segment, $B_{i}$, to project to this point (see Figure 9 .c). Indeed, it can be seen as a extremal subcase of case 1 . 
We can construct simultaneously all these segments with projection vectors on $\mathbb{R}^{2}$ without disturbing one to each other, obtaining a reflecting PBS (see Figure 10 for a complete construction for PAM in Figure 8).

a)

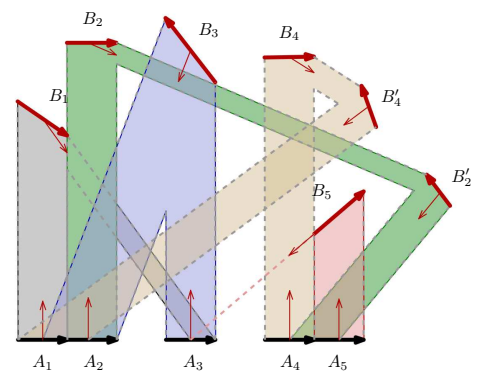

b)

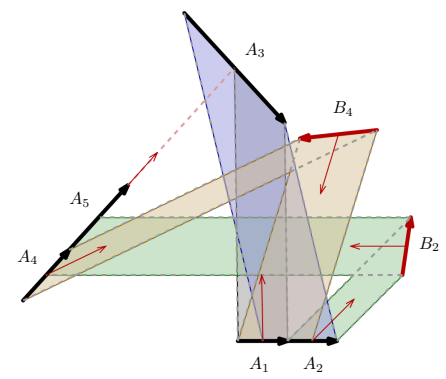

Fig. 10. a) Reflecting PBS simulating PAM b) PBS with reduced number of segments

In order to prove the dynamical simulation, let us consider the following injection between the set of points of the systems:

$$
\begin{aligned}
\varphi: \bigcup I_{i} & \hookrightarrow \bigcup_{i} A_{i} \\
x & \mapsto(x, 0)
\end{aligned}
$$

and the same injection between the dynamics as in the previous theorems.

From the construction, it is obvious that the resulting PBS simulates the given PAM (note that, again, we use only a part of the dynamics of the PBS, considering only the dynamics on the segments $A_{i}$, and not the evolution of the points through the other segments, necessary for the correct computing of the evolution, but not for the dynamics itself).

From above construction, we obtain an upper bound to the number of segments we need in a reflecting PBS to simulate a PAM.

Corollary 1. Let $f$ be a PAM with $N$ affine functions. Let $R$ be the number of affine maps, $f_{i}$, with $a_{i}<0$. Then, there is a reflecting PBS simulating $f$ using, at most, $2 N+R$ reflecting segments.

Remark 4. The method of construction presented previously is not efficient in general, but it works for any possible PAM. In a number of PAM's, it is possible to reduce the number of elements of the PBS simulating the PAM. For example, in Figure 10 .a, we can identify segment $A_{3}$ with $B_{3}$ (of course, taking $A_{3}$ out of the $X$-axis), making unnecessary the use of $B_{1}, B_{3}$ and $B_{5}$. Also, in this example, if we change the orientation of $A_{4}$ and $A_{5}$ we can avoid the use of some auxiliary segments, $B_{2}^{\prime}$ and $B_{4}^{\prime}$. We have reduced the construction from 12 segments to only 7 (it is easy to check that in this example we need, at least, 5 segments in order to simulate the dynamics), see Figure 10 .b. 
Since $\mathbb{Q}$ is closed under linear rational transformations, if we restrict segments and vectors in $\mathbb{Q}^{2}$ for the PBS, and intervals and coefficients in $\mathbb{Q}$ for the PAMs, everything can be proved in the same way and the equivalence remains true.

\section{Unpredictability in rational piecewise maps}

In this section we show that the reachability problem in one dimensional rational piecewise maps is undecidable since for every Minsky machine [9] we can define a PRM that simulates its computation. Actually we need to show how the states, transition function and updates of integer counters can be simulated by a piecewise rational map $P$.

We found a new way how to create a copy of information for a temporal use in dimension one by means of rational functions. It allows us to simulate (in direct way) a Minsky machine in one-dimensional piecewise rational maps (PRM) of degree 2. Note that one-dimensional piecewise affine maps is a subclass of PRMs.

Let $A$ be a 2 -counter machine with a set of states $S=\{1,2, \ldots, n\}$. The configuration of $A$ is a triple $[k, l, s]$ where $k$ and $l$ are values of two counters and $s$ is a current state of $A$. Let us define the mapping $\phi: \mathbb{N} \times \mathbb{N} \times \mathbb{N} \rightarrow \mathbb{Q}$ that is an isomorphism between a configuration $[k, l, s]$ of $A$ and a rational number $s+\frac{1}{2^{k+1} 3^{l+1}}$ that is shifted to the interval $(0,1)$

$$
\phi([k, l, s]) \rightarrow \frac{1}{10^{H}}\left(s+\frac{1}{2^{k+1} 3^{l+1}}\right), H=\lceil\lg (|S|)\rceil
$$

Instead of classical Minsky machine from now on we will consider a wellknown equivalent model of two counter machine where one of the counters is used as a scratchpad. Another, counter holds an integer whose prime factorization is $2^{c} \cdot 3^{d}$. The exponents $c, d$ can be thought of as two virtual counters that are being simulated. If the real counter is set to zero then incremented once, that is equivalent to setting all the virtual counters to zero. If the real counter is doubled, that is equivalent to incrementing $c$, and if it is halved, that is equivalent to decrementing $c$. By a similar procedure, it can be multiplied or divided by 3 , which is equivalent to incrementing or decrementing $d$.

To check if a virtual counter such as $c(d)$ is equal to zero, just divide the real counter by 2 (3), see what the remainder is, then multiply by 2 (3) and add back the remainder. That leaves the real counter unchanged. The remainder will have been nonzero if and only if $c(d)$ was zero.

Let $A$ be in configuration $[k, l, s]$ and it is represented by a number

$$
x=\frac{1}{10^{H}}\left(s+\frac{1}{2^{k+1} 3^{l+1}}\right) .
$$

Let us show that we can perform the operations of multiplication and division by 2 and 3 in a piecewise rational map $P$. To multiply/divide virtual counter by 2 or/and 3 we can use the following expression for $x$, where $a, b$ are integers:

$$
\frac{\left(10^{H} x-s\right) 2^{a} 3^{b}+s}{10^{H}}
$$


Now, we construct a system of intervals with rational functions, associated to them, that allows us to check divisibility of the value of the virtual counter by 2 and 3 or in other words to perform a zero testing on counters of original Minsky machine. For each state $s$ of a counter machine we define the following intervals and functions:

Let us assume that the current configuration $[k, l, s]$ of a machine $M$ is represented by a rational number $x$. If $M$ is in a state $s$ then $x$ belongs to the interval $\left[\frac{s}{10^{H}}, \frac{s+1}{10^{H}}\right]$. Assuming that we know the current state we can add to $x$ an integer $2^{k+1} 3^{l+1}$ by expression $\frac{1}{\left(10^{H} x-s\right)}+x$. In fact for further simulation of checking the emptiness of the one Minsky machine counter we would need to add an integer $2^{k} 3^{l+1}$ using the expression $\frac{1}{2\left(10^{H} x-s\right)}+x$. Such operation gives us an extra information about the counter values in integer part of the number. It is important that we can use it now for some temporal computations and keep another copy of the current state and counter values in the decimal part of the number.

Now we can easily check whether a virtual counter is divisible by 2 iteratively applying $x-2$ while the point $x$ is in the interval $[3,+\infty)$. Finally a point $x$ should reach either the interval $[2,3]$, which corresponds to $k \neq 0$, or the interval $[1,2]$, which corresponds to $k=0$.

In a similar way we can check divisibility by 3 from a state $s$ using negative numbers. If $x \in\left[\frac{s}{10^{H}}, \frac{s+1}{10^{H}}\right]$ we apply $-\left(\frac{1}{3\left(10^{H} x-s\right)}+x\right)$ and then $x+3$ for any point in the interval $(-\infty,-4]$. Next the number $x$ should appear in the interval $[-4,-3]$, which corresponds to $l \neq 0$ or in the interval $[-3,-1]$, which corresponds to $l=0$.

Now we define a piecewise rational map to simulate all operations of Minsky machine such as state transitions, update of counters and testing them for zero. Initially let us define two intervals for intermediate computations related to the zero testing in counters:

If $x \in[3,+\infty)$ then apply $x-2$, If $x \in(-\infty,-4]$ then apply $x+3$

Next for every command of the Minsky machine

State $s$ : IF $k \neq 0$ THEN $\mathrm{k}=\mathrm{k}+\mathrm{a}, \mathrm{l}=\mathrm{l}+\mathrm{b}$ GOTO State $t$ ELSE GOTO State $p$

we define a set of intervals with assigned rational functions:

$$
\begin{aligned}
& \text { If } x \in\left[\frac{s}{10^{H}}, \frac{s+1}{10^{H}}\right] \text { then apply } \frac{1}{2\left(10^{H} x-s\right)}+x \\
& \text { If } x \in\left[2+\frac{s}{10^{H}}, 2+\frac{s+1}{10^{H}}\right] \text { then apply } \frac{\left(10^{H}(x-2)-s\right) \cdot 2^{a} 3^{b}+t}{10^{H}} \\
& \text { If } x \in\left[1+\frac{s}{10^{H}}, 1+\frac{s+1}{10^{H}}\right] \text { then apply } \frac{\left(10^{H}(x-1)-s\right)+p}{10^{H}}
\end{aligned}
$$


where $a \in \mathbb{Z}$ stands for increasing (decreasing) of the first counter by an integer $a$, and $b \in \mathbb{Z}$ stands for increasing (decreasing) of the second counter by an integer $b$.

Next for every command of the Minsky machine with testing of the second counter for zero

$$
\text { State } s \text { : IF } l \neq 0 \text { THEN } \mathrm{k}=\mathrm{k}+\mathrm{a}, \mathrm{l}=\mathrm{l}+\mathrm{b} \text { GOTO State } t \text { ELSE GOTO State } p
$$

We define a set of intervals in a similar way:

$$
\begin{aligned}
& \text { If } x \in\left[\frac{s}{10^{H}}, \frac{s+1}{10^{H}}\right] \text { then apply }-\left(\frac{1}{3\left(10^{H} x-s\right)}+x\right) \\
& \text { If } x \in\left[-\left(3+\frac{s}{10^{H}}\right),-\left(3+\frac{s+1}{10^{H}}\right)\right] \text { then apply } \frac{\left(10^{H}(x+4)-s\right) \cdot 2^{a} 3^{b}+t}{10^{H}} \\
& \text { If } x \in\left[-\left(2+\frac{s}{10^{H}}\right),-\left(2+\frac{s+1}{10^{H}}\right)\right] \text { then apply } \frac{\left(10^{H}(x+3)-s\right)+p}{10^{H}} \\
& \text { If } x \in\left[-\left(1+\frac{s}{10^{H}}\right),-\left(1+\frac{s+1}{10^{H}}\right)\right] \text { then apply } \frac{\left(10^{H}(x+2)-s\right)+p}{10^{H}}
\end{aligned}
$$

Since the computation of a Minsky machine can be simulated by a specially designed PRM the following theorem holds:

Theorem 4. One-dimensional piecewise rational map with a finite number of intervals is the universal model of computations.

Corollary 2. The reachability problem (Problem 1) for one-dimensional PRM is undecidable.

Corollary 3. There exists a particular one-dimensional PRM, that corresponds to the universal Minsky machine, for which the point-to-point reachability problem is undecidable.

\section{Conclusion}

In this paper we show that the model of one-dimensional PAMs is equivalent to a known model of strange billiards from bifurcation and chaos theory. On the other hand we show that predictability in more general one-dimensional class of functions (that includes one-dimensional PAMs) is not possible since we can encode a universal model of computation such as Minsky Machine.

It would be interesting to investigate a natural class of one-dimensional linear rational maps that is in between affine and rational maps. As far as we know the reachability problem for piecewise or nondeterministic maps is open in both cases. The reachability in piecewise linear rational maps related to parameterized reachability in two-dimensional linear maps and the reachability in nondeterministic linear rational maps can be interpreted as parameterized vector reachability problem in $2 \times 2$-matrix semigroups. 


\section{References}

1. Asarin, E.; Maler, O.; Pnueli, A. Reachability analysis of dynamical systems having piecewise-constant derivatives, Theoretical Computer Science 138, (1995) 35-65

2. Asarin, E.; Schneider, G. Widening the boundary between decidable and undecidable hybrid systems. CONCUR'2002,LNCS 2421 (2002) 193-208

3. Blondel, V.; Bournez, O.; Koiran, P.; Papadimitriou, C.; Tsitsiklis, J.: Deciding stability and mortality of piecewise affine dynamical systems. Theor. Comput. Sci. 255(1-2) (2001) 687-696

4. Blondel, V.; Tsitsiklis, J. A survey of computational complexity results in systems and control, Automatica 36 (2000) pp. 1249-1274.

5. Chase, C.; Serrano, J.; Ramadge, P.J. Periodicity and chaos from switched flow systems:contrasting examples of discretely controlled continuous systems. IEEE Trans. Automatic Control, 38 (1993) 70-83

6. Koiran, P.; Cosnard, M.; Garzon, M. Computability with Low-Dimensional Dynamical Systems. Theor. Comput. Sci. 132(2) (1994) 113-128.

7. Koiran, P. The topological entropy of iterated piecewise affine maps is uncomputable. DMTCS 4(2), (2001) 351-356.

8. Kurganskyy, O.; Potapov, I.. Computation in One-Dimensional Piecewise Maps and Planar Pseudo-Billiard Systems. Unconventional Computation, LNCS 3699, 169$175,2005$.

9. Minsky, M. Computation: Finite and Infinite Machines, Prentice-Hall, Inc., N.J., 1967.

10. Peters, K.; Parlitz, U. Hybrid systems forming strange billiards. Int. J. of Bifurcations and Chaos, 19, (2003) 2575-2588.

11. Schurmann, T.; Hoffman, I. The entropy of strange billiards inside n-simplexes. J. Phys. A 28, (1995) 5033-5039. 